\title{
Ultrastructural Features of Eggshells of the Green Turtles, Chelonia mydas from Ras Al-Hadd Reserve, Oman
}

\author{
T. A. Ba-Omar ${ }^{*}$, S. N. Al-Bahry ${ }^{*}$, I. Al-Amri** A.Y. Al-Kindi ${ }^{*}$ and I.Y. Mahmoud ${ }^{*}$ \\ *Department of Biology, College of Science, Sultan Qaboos University, P.O.Box 36 \\ Muscat 123, Sultanate of Oman. \\ ** Department of Pathology, EM Unit, College of Medicine, Sultan Qaboos \\ University
}

Few studies were carried out on the structure of the eggshells of the sea turtles [1]. This is the first study on the ultrastructure of the eggshells of the green turtles Chelonia mydas in Oman

Eggs of green sea turtles, Chelonia mydas were collected from Ras Al-Hadd. Eggshells were taken from freshly oviposited eggs and from eggs soon after hatching. Samples were cleaned and cut into small pieces. Some eggshells were examined under low vacuum condition (untreated) and viewed with back scattered electron detector (BSE) at $20 \mathrm{kV}$ and $20 \mathrm{~Pa}$ vacuum pressure using JEOL JSM-5600LV, SEM. Others were examined under high vacuum condition after being gold coated (BioRad SEM Sputter coater) using JSM-5600LV, SEM and viewed with secondary electron detector $(\mathrm{SE})$ at $5 \mathrm{kV}$.

Eggshell consists of three layers: an outer layer, inner layer and eggshell membrane (Fig. 1a). The outer layer is predominately made up of loose calcium carbonate crystals arranged in radiating forms around the pores (Fig. 1a, b). Various shapes and sizes of crystallites are poorly arranged and loosely connected with each other, forming between them large irregular pores which serve for gas exchange. Some crystals are in the form of spicules radially arranged around pores while others are shaped like platelets or globules (Fig. 1a, b). Some of the platelets are porous resembling honeycombs (Fig. 1a). The inner layer is tightly compacted with crystallites divided into small sections. Each section consists of a small central pore surrounded by strata of rod-shaped crystals arranged in a longitudinal axis to each pore (Fig. 1d, e). Large number of these sections makes a larger section. These large sections unite with each other forming irregular large pores or grooves (Fig.1d, e). The inner layer communicates with the outer layer and with the eggshell membrane by a network of rod-shaped crystals running at different angles (Fig. 1a, d). The inner shell membrane is made up of a thin sheet contains numerous networks of fibers (Fig.1 c). There are numerous pores in the outer and inner layers and they continue into the eggshell membrane. Apparently, these pores are for the purpose of gas exchange. Generally, there is no radical changes in the eggshell structures before or after incubation. However, hatched eggshells showed that some large crystals are lost from the outer layer during the process of incubation leaving bare spot on this layer and exposing the inner layer directly to the sand (Fig. 1f). This is probably caused by either expansion of the shell from sand moisture absorption or friction between the outer layer and the sand due to the movements of the embryo during the incubation period.

Reference:

[1] G. Saho et al.. (1996). Acta Anat. 156:261-267 


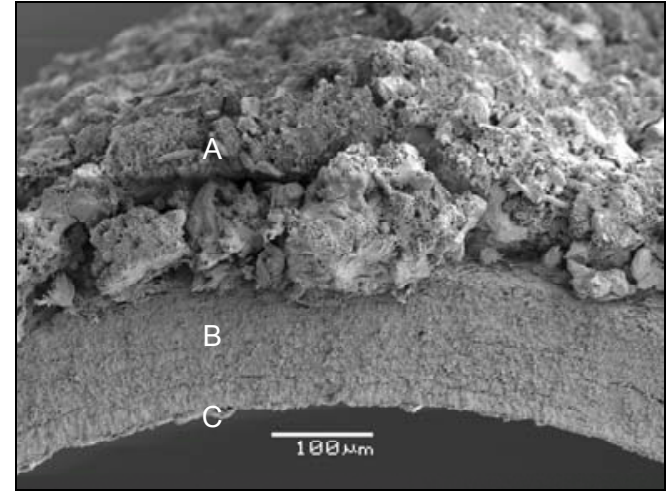

a



$\mathrm{c}$

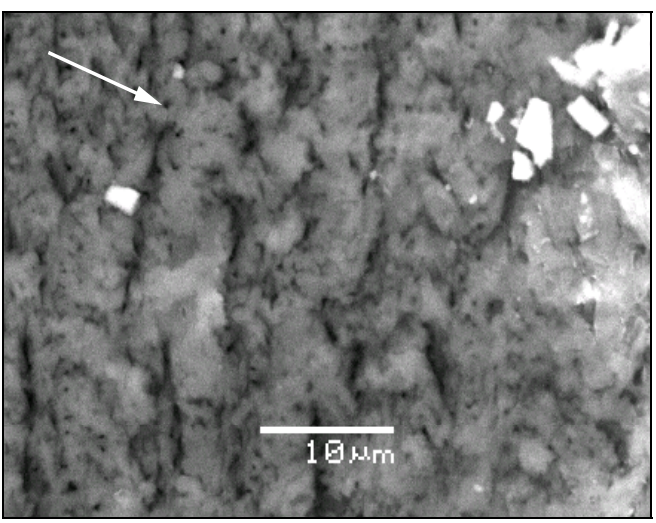

e

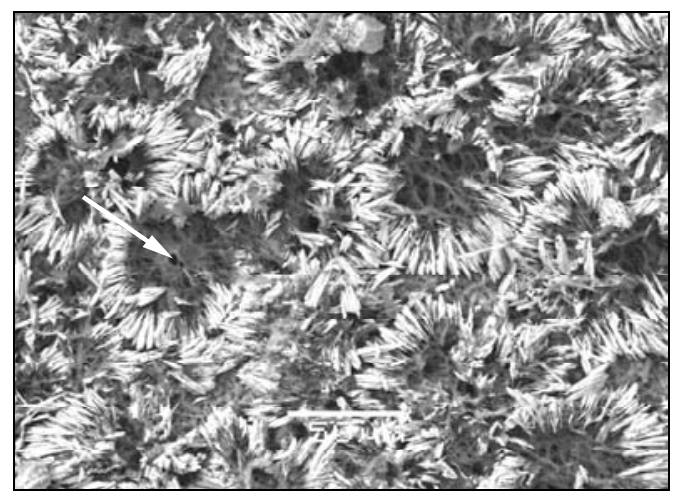

$\mathrm{b}$

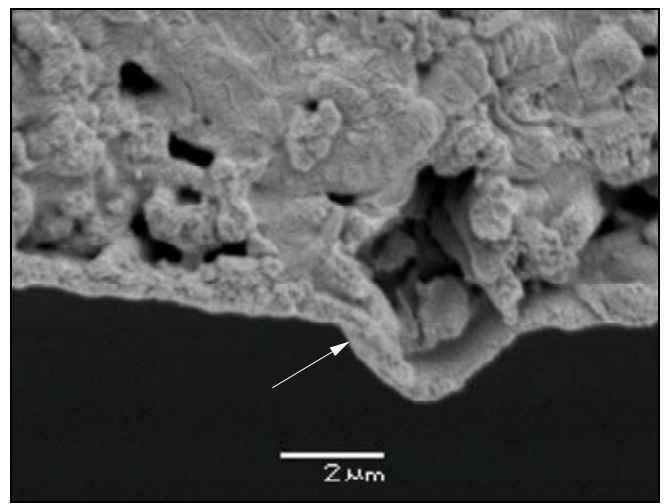

d

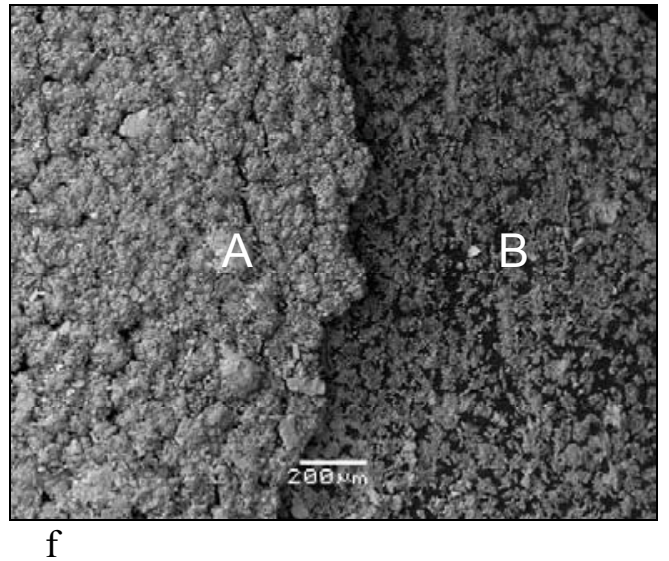

Fig 1. Eggshell components of green turtle, Chelonia mydas

$\mathrm{a}=$ The three layers of egg shell $(\mathrm{A}=$ outer layer, $\mathrm{B}=$ inner layer, $\mathrm{C}=$ eggshell membrane $)$; note the loose crystals, some are porous like honeycomb (A), inner layer is more compacted and organized (B) and shell membrane is thin (C).

$\mathrm{b}=$ Radiating spicules $(\rightarrow)$, a typical feature of the outer layer.

$\mathrm{c}=$ Eggshell membrane, note the network of fibers and the numerous pores which communicate inside the egg for gas exchange.

$\mathrm{d}=$ The inner layer arranged in units. Each unit consists of rod shape crystals arranged in longitudinal axes around the small pores $(\rightarrow)$.

$\mathrm{e}=\mathrm{A}$ higher magnification view of figure (d).

$\mathrm{f}=\mathrm{A}$ hatched eggshell. Notice the lost of outer layer (A) and exposure of inner layer (B). 